\title{
Obscure gastrointestinal bleeding: a complication of radiation enteritis diagnosed by wireless capsule endoscopy
}

\author{
D. Martínez Ares, B. González Conde, J. Souto Ruzo, M. A. Vázquez Millán, E. Estévez Prieto, \\ P. Alonso Aguirre and J. L. Vázquez Iglesias
}

Division of Gastroenterology. Complejo Hospitalario Universitario Juan Canalejo. A Coruña, Spain

\begin{abstract}
Obscure gastrointestinal bleeding is a common disorder and may account for as many as $5 \%$ of all gastrointestinal hemorrhages. It is often caused by lesions in the small intestine, which were very complicated to examine prior to the advent of wireless capsule endoscopy.

Here we present the case of a 31-year-old woman with obscure gastrointestinal bleeding as a complication of radiation enteritis, which was diagnosed only after she underwent an examination with wireless capsule endoscopy.

This technique has proven to be far superior to other radiographic and endoscopic methods in diagnosing obscure gastrointestinal bleeding and pathologies of the small intestine in general.
\end{abstract}

Key words: Radiation enteritis. Gastrointestinal bleeding. Obscure gastrointestinal bleeding. Capsule endoscopy.

Martínez Ares D, González Conde B, Souto Ruzo J, Vázquez Millán MA, Estévez Prieto E, Alonso Aguirre P, Vázquez Iglesias JL. Obscure gastrointestinal bleeding: a complication of radiation enteritis diagnosed by wireless capsule endoscopy. Rev Esp Enferm Dig 2004; 96: 132-137.

\section{INTRODUCTION}

The concept of obscure gastrointestinal bleeding covers all causes of GI bleeding that fail to be clarified after performing conventional radiographic and endoscopic tests. In some series they account for as many as $5 \%$ of all cases of GI bleeding $(1,2)$. These hemorrhages may be occult, their only manifestation being chronic anemia and a positive result on tests for occult blood in the feces, or

Recibido: 21-04-03.

Aceptado: 28-04-03.

Correspondencia: David Martínez Ares. C/ Emilio González López, 11, $7^{\circ}$ B. 15011 A Coruña. e-mail: dmartinez@sepd.es they may be overt, appearing as melena or rectorrhage (3). In a large number of cases bleeding originates in the small intestine, a region that is difficult for conventional endoscopy to access. Moreover, radiological studies, which are currently the only procedures that permit the examination of the small intestine, are unable to show small bleeding lesions such as angiodysplasias, which are one of the most common causes of obscure gastrointestinal bleeding $(4,5)$.

With the advent of wireless capsule endoscopy great progress has been made in the study of these portions of the intestine, which have remained unexplored until now. The capsule makes it possible to provide an etiological diagnosis of obscure origin bleeding in 33-100\% (6) of cases. In a study compiling the 517 cases published to date, 343 patients $(65 \%)$ could be diagnosed with wireless capsule endoscopy (7).

Following is a case report of a patient with obscure gastrointestinal bleeding associated with a relatively common pathology, but whose diagnosis was only possible after she was examined with this novel endoscopic technique.

\section{CASE REPORT}

A 31-year-old patient presented to our hospital on two occasions with symptoms of severe anemia. On her second hospital admission, she also exhibited melenic stools. A blood test done on admission showed a hemotocrit value of $24 \%$ (normal value is $36-45 \%$ ) and a hemoglobin level of $8 \mathrm{~g} / \mathrm{dL}(12-15.6 \mathrm{~g} / \mathrm{dL})$.

When questioned about her personal background, she reported that eight years earlier a neoplasm of the uterus had been diagnosed and treated with chemotherapy and pelvic radiotherapy. Since that time she had been asymptomatic, with no evidence of a recurrent tumor in followup controls. 
It was decided to perform a gastroscopy, which did not reveal any lesions that could explain her symptoms. Afterward the patient underwent a colonoscopy with ileoscopy, push enteroscopy, and barium examination of the small bowel, all of which had negative results. For this reason we recommended that the patient be examined with wireless capsule endoscopy, since she presented no apparent contraindications. A study of the images revealed the presence of granular, edematous areas in the mucosa, with ulcerated zones producing a slight stenosis at the level of the ileum (Fig. 1) and telangiectasias that bled spontaneously on contact (Fig. 2). Considering the patient's history of pelvic radiotherapy and an unlikely diagnosis of Crohn's disease, our findings strongly suggested radiation enteritis. The ingestion of non-steroid anti-inflammatory medication had also been previously ruled out.

Our recommendation to the patient was surgical treatment. She rejected this possibility, at least for the time being, so we started her on treatment with corticosteroids and 5-ASA. Barring the persistence of a certain degree of anemia, the patient's condition has improved somewhat, and she has not required any more transfusions.

\section{DISCUSSION}

Radiation enteritis generally affects the distal ileum, sigmoid colon and rectum (8) owing to their proximity to or their location in the pelvic region, since gynecological neoplasias and prostate cancer are the diseases most frequently treated with radiotherapy.

The damage induced by radiation may be acute, subacute or chronic. Acute lesions are usually due to damage to the epithelium (9), motor disorders from neural irritation, and other anomalies caused by radiation $(10,11)$. Symptoms usually occur a few days or weeks after exposure. Chronic lesions, however, are due to an ischemia in the wall of the intestine caused by a collagenous-vascular lesion. They may appear as strictures, chronic ulcerations, lower gastrointestinal bleeding (occult or overt), perforations of the intestine, formation of fistulous passages or intra-abdominal abscesses, etc. (12). The latency period of these manifestations may range from 6 months to 25 years (13). Our patient presented no evidence of previous symptoms, and eight years had passed since she was last treated with radiation.

In the chronic form of the disease medical treatment is often ineffective, with surgery being required to treat complications $(14,15)$. Our efforts should therefore focus on prevention, either with medication that protects against radiation or anti-inflammatory treatment (16). Also helpful would be the use of absorbable mesh slings which separate the intestine from the field of radiation (17). Our patient rejected surgical treatment after she was given the diagnosis. However, when an evaluation with wireless capsule endoscopy was suggested, she was willing to accept the fact that it might be necessary. If she

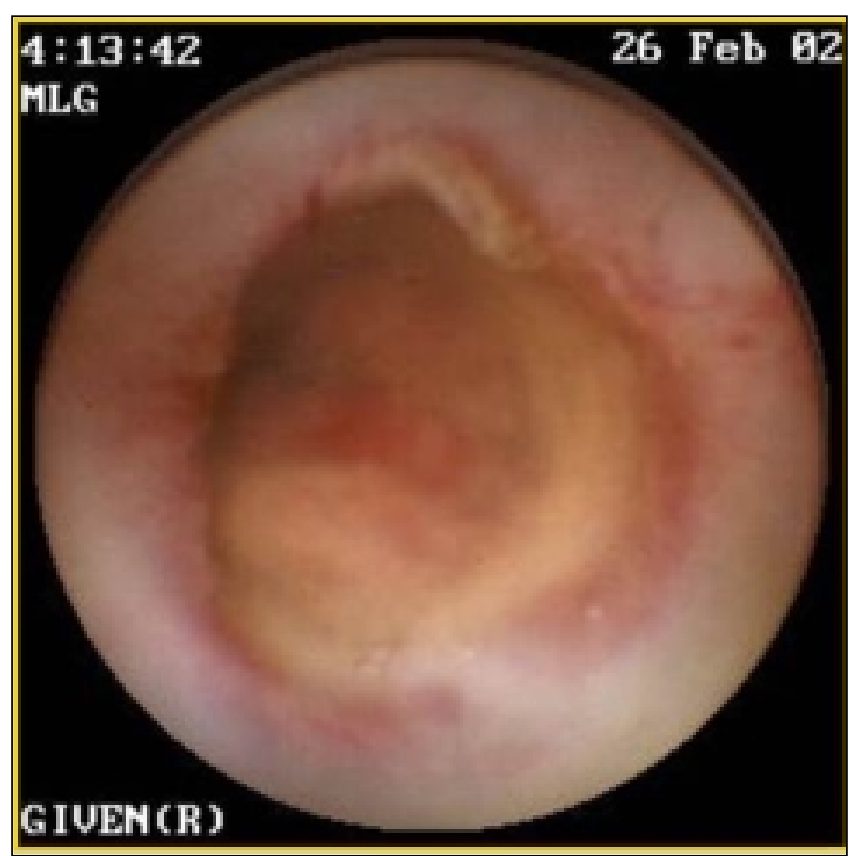

Fig. 1.- This image shows ulcerated areas causing moderate stenosis, and the adjacent mucosa has a bare appearance

En la imagen se aprecian áreas ulceradas que producen una discreta estenosis, con la mucosa adyacente con aspecto denudado.

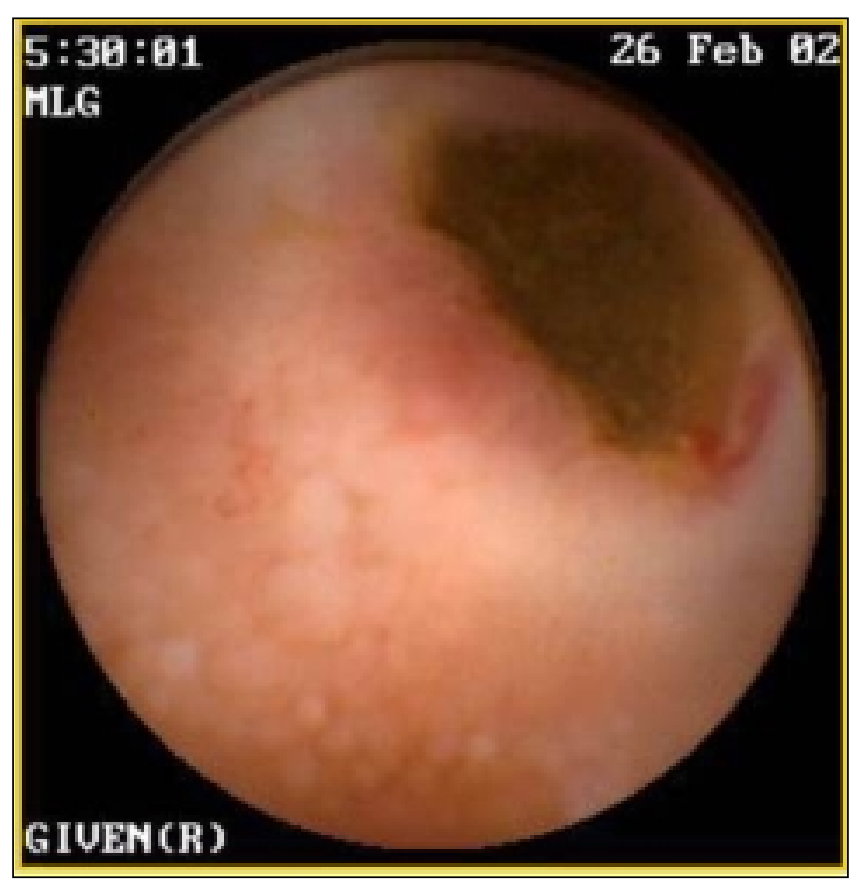

Fig. 2.- Photograph showing newly formed vessels, which bleed spontaneously on contact

En la fotografía se aprecian además vasos neoformados, que presentan sangrado espontáneo y al roce.

had refused, the test would have been ruled out, since, as we will discuss below, it is absolutely essential for the patient to be comfortable with this possibility. 
Images provided by the capsule were evaluated as highly suggestive of radiation enteritis, particularly in view of the patient's history. The ulcerated areas, the dull appearance of the mucosa, and the constricted areas might be attributed to lesions caused by non-steroid antiinflammatory medication or even resemble lesions from Crohn's disease, which has been referred to as a cause in as many as $10-20 \%$ of the cases of obscure gastrointestinal bleeding (18). The patient professed not to have taken non-steroid anti-inflammatories, and Crohn's disease was ruled out owing to the undamaged state of the terminal ileum and the absence of lesions in other areas. Moreover, the presence of the characteristic telangiectasias in the mucosa of the intestine would strongly suggest a possible diagnosis of radiation enteritis, although we could not back it up with histological confirmation.

There is little doubt that the main application of wireless capsule endoscopy today is the study of obscure gastrointestinal bleeding. This technique has also proved to be far superior to push enteroscopy in diagnosing these hemorrhages. Several other studies also corroborate these findings, although only three reported statistically significant results (7). Two have been published as abstracts $(19,20)$, while only one has been published as a fulllength article (21). In this study, Ell et al. reported that push enteroscopy resulted in a diagnosis in $28 \%$ of cases, whereas the percentage with wireless capsule endoscopy rose to $66 \%(\mathrm{p}<0.001)$.

In virtue of the AGA recommendations on the management of obscure gastrointestinal bleeding (22), routine endoscopic examinations must first be performed before resorting to the capsule. Following this pattern, our patient underwent a gastroscopy, colonoscopy, enteroscopy, and barium examination of the small bowel, all of which had a negative outcome. Not only did the wireless capsule endoscopy reveal lesions prone to bleeding in the small intestine, it also located active bleeding in some of these lesions.

Wireless capsule endoscopy might also prove useful in the study of other pathologies of the small intestine besides obscure gastrointestinal bleeding, where other endoscopic techniques have failed to result in a diagnosis (23). Under these circumstances it clearly outranks barium radiology $(24)$.

In addition, an examination using wireless capsule endoscopy is an extremely safe, non-invasive and painless procedure that may be performed on an out-patient basis (23), provided that the patients have been properly screened. The test is not recommended in patients suspected of suffering from stenosis or intestinal obstruction, or in patients having difficulty swallowing or with severe motor disorders of the gastrointestinal tract. It should not be administered if the patient is unable to understand the explanation of the test, and hence, will not be able to collaborate, and in cases where the patient wears an electronic device such as a pacemaker, etc. (25). There are no studies on the use of this test in children under the age of 10 . The only absolute contraindication, however, is when the patient clearly exhibits a negative attitude towards surgery or if a surgical procedure would entail an unacceptable risk (26), as surgery may be required to disimpact the device.

In summary, wireless capsule endoscopy has remedied, in part, the grave deficiencies in the techniques that were available to carry out the examination of the small intestine, although roughly $35 \%$ of patients with obscure gastrointestinal bleeding will still remain undiagnosed. This technique is safe, with practically no complications at all when performed on patients who have been correctly screened. This non-invasive test has been widely accepted by patients.

\section{REFERENCES}

1. Szold A, Katz L, Lewis B. Surgical approach to occult gastrointestinal bleeding. Am J Surg 1992; 163: 90-3.

2. Adrain AL, Krevsky B. Enteroscopy in patiens with gastrointestinal bleeding of obscure origin. Dig Dis Sci 1996; 14: 345-55.

3. Zuckerman GR, Prakash C, Askim MP, et al. AGA technical review on the evaluation and management of occult and obscure gastrointestinal bleeding. Gastroenterology 2000; 118: 201-21.

4. Douard R, Wind P, Panis Y, et al. Intraoperative enteroscopy for diagnosis and management of unexplained gastrointestinal bleeding. Am J Surg 2000; 180: 181-4.

5. Nolan DJ, Traill ZC. The current role of the barium examination of the small intestine. Clin Radiol 1997; 52: 809-20.

6. Appleyard M, Glukhovsky A, Swain P. Wireless-capsule diagnostic endoscopy for recurrent small-bowel bleeding. N Engl J Med 2001; 344 (3): 232-3.

7. Caunedo Álvarez A, Rodríguez Téllez M, Barroso Relinque N, García Montes JM, Pellicer Bautista FJ, Herrerías Gutiérrez JM. Role of capsule endoscopy in the management of patients with obscure gastrointestinal bleeding. Rev Esp Enferm Dig 2002; 94: 482-7.

8. Yoshimura K, Hirata I, Maemura K, Sugi K, Tahara T. Radiation enteritis: a rare complication of the transverse colon in uterine cancer. Intern Med 2000; 39 (12): 1060-3.

9. MacNaughton WK. Review article: new insights into the pathogenesis of radiation-induced intestinal dysfunction. Aliment Pharmacol Ther 2000; 14 (5): 523-8.

10. Classen J, Belka C, Paulsen F, Budach W, Hoffmann W, Bamberg M. Radiation-induced gastrointestinal toxicity. Pathophysiology, approaches to treatment and prophylaxis. Strahlenther Onkol 1998; 174 (Supl. 3): 82-4.

11. Fraser R, Frisby C, Schirmer M, Blackshaw A, Langman J, Yeoh E, et al. Effects of fractionated abdominal irradiation on small intestinal motility: studies in a novel in vitro animal model. Acta Oncol 1997; 36 (7): 705-10.

12. Nguyen NP, Antoine JE. Radiation enteritis. In: Feldman M, Friedman LS, Sleisenger MH, eds. Sleisenger and Fordtran's, Gastrointestinal and Liver Disease. Philadelphia: Saunders, 2002. p. 1996-7.

13. Nguyen NP, Antoine JE. Radiation enteritis. In: Feldman M, Friedman LS, Sleisenger MH, eds. Sleisenger and Fordtran's, Gastrointestinal and Liver Disease. Philadelphia: Saunders, 2002. p. 1998.

14. Regimbeau JM, Panis Y, Gouzi JL, Fagniez PL. Operative and long term results after surgery for chronic radiation enteritis. Am J Surg 2001; 182 (3): 237-42.

15. Pagano A, Vaccaro F, Romano G, Romano G Surgical treatment of radiation enteritis. Minerva Chir 1996; 51 (3): 93-6.

16. Kilic D, Eeghan I, Ozenirler S, Dursun A. Double-blinded, randomized, placebo-controlled study to evaluate the effectiveness of sulphasalazine in preventing acute gastrointestinal complications due to radiotherapy. Radiother Oncol 2000; 57 (2): 125-9.

17. Rodier JF, Janser JC, Rodier D, Dauplat J, Kauffmann P, Le Bouedec G, Giraud B, Lorimier G. Prevention of radiation enteritis by an absorbable polyglycolic acid mesh sling. A 60 -case multicentric study. Cancer 1991; 68 (12): 2545-9. 
18. Hahne M, Adamek HE, Schilling D, Riemann JF. Wireless capsule endoscopy in a patient with obscure occult bleeding. Endoscopy 2002; 34 (7): 588-90.

19. Gay G, Saurin JC, Gaudin JL, et al. Comparison of wireless endoscopic capsule and push-enteroscopy in patients with obscure ocult/overt digestive bleeding: results of a prospective, blinded, multicenter trial. First Given conference on capsule endoscopy. Rome, 2002. p. 17-9.

20. Pennazio M, Santucci R, Rondonotti E, et al. Wireless capsule endoscopy in patients with obscure gastrointestinal bleeding: results of the italian multicentre experience. First Given conference on capsule endoscopy. Rome, 2002. p. 17-9.

21. Ell C, Remke S, May A, Helou L, Henrich R, Mayer G. The first prospective controlled trial comparing wireless capsule endoscopy with push enteroscopy in chronic gastrointestinal bleeding. Endoscopy 2002; 34 (9): 685-9.
22. American Gastroenterological Association medical position statement. Evaluation and management of occult and obscure gastrointestinal bleeding. Gastroenterology 2000; 118: 197-200.

23. Scapa E, Jacob H, Lewkowicz S, Migdal M, Gat D, Gluckhovski A, et al. Initial experience of wireless-capsule endoscopy for evaluating occult gastrointestinal bleeding and suspected small bowel pathology. Am J Gastroenterol 2002; 97 (11): 2776-9.

24. Costamagna G, Shah SK, Riccioni ME, Foschia F, Mutignani M, Perri $\mathrm{V}$, et al. A prospective trial comparing small bowel radiographs and video capsule endoscopy for suspected small bowel disease. Gastroenterology 2002; 123 (4): 999-1005.

25. Barroso Relinque N, Caunedo Álvarez A, Rodríguez Téllez M, Herrerías Estéban JM, Pellicer Bautista F, Herrerías Gutiérrez JM. Wireless endoscopy. Rev Esp Enferm Dig 2001; 93: 598-601.

26. Cave DR. Wireless Video Capsule Endoscopy. Clinical Perspectives in Gastroenterolgy 2002: 203-7.

\title{
Hemorragia digestiva de origen oscuro secundaria a enteritis actínica: diagnóstico por cápsula endoscópica
}

\author{
D. Martínez Ares, B. González Conde, J. Souto Ruzo, M. A. Vázquez Millán, E. Estévez Prieto, \\ P. Alonso Aguirre y J. L. Vázquez Iglesias
}

Servicio de Aparato Digestivo. Complejo Hospitalario Universitario Juan Canalejo. A Coruña

\section{RESUMEN}

La hemorragia digestiva de origen oscuro es una entidad frecuente pudiendo representar hasta un $5 \%$ del total de las hemorragias digestivas. Su origen se encuentra en muchas ocasiones en lesiones en el intestino delgado, cuya exploración era muy complicada antes de la aparición de la cápsula endoscópica.

Presentamos el caso de una mujer de 31 años con una hemorragia digestiva de origen oscuro secundaria a una enteritis actínica que sólo pudo ser diagnosticada tras ser sometida a la exploración con cápsula endoscópica.

Esta técnica se ha mostrado claramente superior a las otras técnicas radiológicas y endoscópicas en el diagnóstico de las hemorragias digestivas de origen oscuro y la patología del intestino delgado en general.

Palabras clave: Enteritis actínica. Hemorragia digestiva. Hemorragia digestiva de origen oscuro. Cápsula endoscópica.

\section{INTRODUCCIÓN}

El concepto de hemorragia digestiva de origen oscuro engloba a todas aquellas causas de sangrado digestivo que no pueden ser esclarecidas con las exploraciones endoscópicas y radiológicas convencionales. En algunas series llegan a representar un 5\% del total de las hemorragias digestivas $(1,2)$. Estos sangrados pueden ser ocultos, manifestándose como anemia y prueba de sangre oculta en heces positiva, o ser un sangrado evidente en forma de melenas o rectorragias (3). En un número importante de casos el origen del sangrado está en el intestino delgado, que resulta poco accesible para la endoscopia convencional (4). Además, los estudios radiológicos, los únicos que nos permitían explorarlo hasta el momento, no son capaces de mostrarnos pequeñas lesiones sangrantes tales como las angiodisplasias, que representan una de las causas más frecuentes de hemorragia de origen oscuro (5).

La aparición de la cápsula endoscópica ha supuesto un gran avance para el estudio de estos tramos de intestino hasta ahora inexplorados. Con la cápsula se puede llegar a un diagnóstico etiológico de la hemorragia de origen oscuro en un 33-100\% (6) de los casos. En un estudio en el que se recogieron los 517 casos publicados hasta el momento, 343 pacientes $(65 \%)$ pudieron ser diagnosticados con la cápsula endoscópica (7).

Presentamos a continuación un caso de hemorragia digestiva de origen oscuro en relación con una patología re- 
lativamente frecuente, pero que sólo pudo ser diagnosticado tras someter a la paciente a una exploración con esta novedosa técnica endoscópica.

\section{CASO CLÍNICO}

Se trata de una paciente de 31 años que ingresa en nuestro centro en dos ocasiones con un cuadro de anemia severa. En el segundo de los ingresos presenta además deposiciones melénicas. El hemograma realizado al ingreso revela un hematocrito del $24 \%$ (valores normales $36-45 \%$ ) y una hemoglobina de $8 \mathrm{~g} / \mathrm{dl}$ (12-15,6 g/dl).

Interrogada sobre sus antecedentes personales refiere que ocho años antes había sido diagnosticada y tratada de una neoplasia uterina con quimioterapia y radioterapia pélvica. Desde entonces había estado asintomática y sin evidencia de recidiva tumoral en los controles posteriores.

Se indica inicialmente una gastroscopia, en la que no se objetiva lesión alguna que justificase el cuadro. Se realizaron posteriormente una colonoscopia con ileoscopia, una enteroscopia de pulsión y un tránsito gastrointestinal baritado, todos ellos con resultado negativo. Por este motivo, propusimos a la paciente la exploración con la cápsula endoscópica, dado que no parecía existir contraindicación alguna. Tras el estudio de las imágenes se objetivó a nivel de íleon, la presencia de áreas de mucosa granular, edematosa, con zonas ulceradas que producían una discreta estenosis (Fig. 1) y telangiectasias que sangraban espontáneamente y al roce (Fig. 2). Todos estos hallazgos, considerando el antecedente de radioterapia pélvica y que una enfermedad de Crohn parecía improbable, nos parecían muy sugestivos de enteritis actínica. Por otra parte, la ingesta de AINE también había sido descartada con anterioridad.

Se propuso tratamiento quirúrgico a la paciente. Se desestimó esta posibilidad, al menos por el momento, por lo que se inició tratamiento con corticosteroides y 5-ASA. Ha experimentado cierta mejoría, si bien es cierto que persiste cierta anemia, aunque no ha vuelto a precisar transfusiones.

\section{DISCUSIÓN}

La enteritis actínica suele afectar el íleon distal, colon sigmoide y recto (8) por su proximidad o localización en la pelvis, dado que son las neoplasias ginecológicas y el cáncer prostático los procesos que con mayor frecuencia reciben tratamiento con radioterapia.

El daño inducido por la radiación puede ser agudo, subagudo o crónico. Las lesiones agudas suelen deberse al daño epitelial (9), los trastornos motores originados por la irritación neural y otras anomalías provocadas por la radiación $(10,11)$. La sintomatología suele aparecer a los pocos días o semanas de la exposición. Las lesiones crónicas, sin embargo, se deben a la isquemia de la pared intestinal provocada por la lesión colágeno-vascular. Pueden mostrarse en forma de estenosis, ulceraciones crónicas, hemorragia digestiva baja (oculta o manifiesta), perforaciones intestinales, formación de trayectos fistulosos, abscesos intraabdominales (12), etc. Estas manifestaciones pueden presentarse con una latencia que puede oscilar desde 6 meses hasta 25 años (13). En nuestra paciente no había constancia de manifestaciones previas, y en el momento actual habían transcurrido ocho años desde la radiación.

En la enfermedad crónica el tratamiento médico es a menudo infructuoso, requiriéndose tratamiento quirúrgico de las complicaciones $(14,15)$. Nuestros esfuerzos deberán centrarse, por lo tanto, en la prevención ya sea con radioprotectores o antiinflamatorios (16), o usando mallas reabsorbibles que alejen el intestino del campo de la radiación (17). Nuestra paciente rechazó el tratamiento quirúrgico una vez se llegó al diagnóstico. Sin embargo, al planteársele la exploración con la cápsula endoscópica sí había asumido la posible necesidad del mismo. En caso contrario se habría contraindicado la prueba, ya que como se comentará más adelante, la aceptación de esta posibilidad es un requisito indispensable.

Las imágenes ofrecidas por la cápsula nos parecieron muy sugestivas de una enteritis por radiación, sobre todo al considerar los antecedentes. Las áreas ulceradas, la mucosa deslustrada y las áreas estenóticas podrían corresponder a lesiones por AINE e incluso semejar lesiones de la enfermedad de Crohn, que también ha sido referido como causa de hasta un 10-20\% de las hemorragias de origen oscuro (18). La paciente negaba la ingesta de AINE y, en lo referente a la enfermedad de Crohn, la indemnidad del íleon terminal y la ausencia de lesiones a otros niveles parecían descartarlo. Por otra parte, la presencia de las características telangiectasias en la mucosa intestinal nos sugirió con mayor fuerza la posibilidad de una enteritis actínica, aunque no disponíamos de la confirmación histológica.

Hay pocas dudas en la actualidad de que la principal indicación de la cápsula endoscópica es el estudio de las hemorragias digestivas de origen oscuro. También parece demostrada la superioridad de la cápsula en sobre la enteroscopia de pulsión en el diagnóstico de las mismas. Hay varios estudios que así lo expresan, aunque sólo tres de ellos obtienen resultados estadísticamente significativos (7). Dos de ellos han sido publicados en forma de abstract (el estudio de Gay y cols. (19) y el de Pennazio y cols. (20), y sólo uno publicado como artículo completo (21). En este estudio, Ell y cols. observan cómo la enteroscopia de pulsión obtiene un diagnóstico en un $28 \%$ de los casos, mientras que la cápsula endoscópica alcanza unas cifras del 66\% ( $\mathrm{p}<0,001)$.

En virtud de las recomendaciones de la AGA en el manejo de las hemorragias digestivas de origen oscuro (22), han de agotarse primero las exploraciones endoscópicas rutinarias antes de recurrir a la cápsula. Siguiendo esta pauta, nuestra paciente fue sometida a gastroscopia, colonosocopia, enterosocopia y tránsito baritado, todos ellos 
con resultado negativo. La cápsula endoscópica no sólo reveló lesiones potencialmente sangrantes en el intestino delgado, sino que también objetivó sangrado activo en alguna de ellas.

La cápsula endoscópica también podría tener utilidad en el estudio de otras patologías del intestino delgado al margen de las hemorragias de origen oscuro, en cuyo diagnóstico hayan fracasado otros medios endoscópicos (23). También en estas circunstancias se muestra claramente superior a la radiología baritada (24).

Además, la exploración con cápsula endoscópica es una técnica poco invasiva, no dolorosa, que se puede realizar en regimen ambulatorio y altamente segura (23), siempre y cuando se seleccionen adecuadamente los pacientes. La exploración está contraindicada cuando se sospechen estenosis u obstrucción intestinal, cuando existen trastornos en la deglución o trastornos motores severos del tracto gastrointestinal, cuando el paciente es incapaz de entender, y por tanto de colaborar, en la prueba, y cuando el enfermo es portador de dispositivos electrónicos como marcapasos, etc. (25). No hay estudios acerca de su utilización en niños menores de 10 años. No obstante, la única contraindicación absoluta viene dada por la negativa manifiesta del paciente a la cirugía o cuando una intervención quirúrgica supone un riesgo inaceptable (26), ya que la impactación del dispositivo puede requerir solución quirúrgica.

En resumen, la cápsula endoscópica ha solucionado en parte las grandes deficiencias que teníamos en la exploración del intestino delgado, aunque aún quedarían aproximadamente un $35 \%$ de pacientes con hemorragias digestivas de origen oscuro sin diagnóstico. Se trata de una técnica segura, exenta prácticamente de complicaciones si se realiza una correcta selección de los pacientes, y además, una técnica poco cruenta, con gran aceptación en los pacientes. 